\title{
Lack of Action Potential-Prolonging Effect of Terfenadine on Rabbit Myocardial Tissue Preparations
}

\author{
Haruko Masumiya, ${ }^{a, b}$ Mariko Saito, ${ }^{a}$ Mie Ito, ${ }^{a}$ Tomoyuki Matsuda, ${ }^{a}$ Kazuo Noguchi, ${ }^{a}$ \\ Naoko IIDA-TANAKA, ${ }^{a, c}$ Hikaru TANAKA, ${ }^{*, a}$ and Koki ShigenOBU ${ }^{a}$ \\ ${ }^{a}$ Department of Pharmacology, Toho University School of Pharmaceutical Sciences; 2-2-1 Miyama, Funabashi, Chiba \\ 274-8510, Japan: ${ }^{b}$ Department of Bio-informational Pharmacology, Medical Research Institute, Tokyo Medical and \\ Dental University; 2-3-10 Kanda Surugadai, Chiyoda-ku, Tokyo 101-0062, Japan: and ${ }^{c}$ Department of Food Science, \\ Otsuma Woman's University; 12 Sanbancho, Chiyoda-ku, Tokyo 102-8357, Japan.
}

Received August 1, 2003; accepted September 17, 2003

The effects of terfenadine, an antiallergic drug also known for its QT-prolonging and arrhythmogenic activities, on the action potential of isolated myocardial tissue preparations from rabbits were examined with microelectrode techniques. In the Purkinje fibers and atrium, terfenadine concentration dependently decreased the maximum rate of rise $\left(+\dot{V}_{\text {max }}\right)$ without affecting other action potential parameters. In the ventricle, terfenadine had little effect on action potential configuration. In the sinoatrial node, terfenadine $20 \mu_{\mathrm{M}}$ prolonged cycle length mainly through inhibition of $+\dot{V}_{\max }$. Terfenadine $1 \mu_{\mathrm{M}}$ completely inhibited the human ether a go-go-related gene (HERG) channel current expressed in HEK293 cells in the same experimental solution as in microelectrode experiments. The lack of terfenadine effect on the action potential duration suggests that there are drugs for which the HERG channel inhibitory action underlying in vivo QT prolongation cannot be evaluated based on their action potential-prolonging activity in isolated myocardial tissue preparations.

Key words terfenadine; QT prolongation; action potential duration; Purkinje fiber; human ether a go-go-related gene

It is well recognized that both cardiovascular and noncardiovascular drugs may promote arrhythmia. ${ }^{1)}$ Clinical treatment with class I and class III antiarrhythmic agents as well as non-cardiovascular drugs such as terfenadine, cisapride, and haloperidol has been associated with QT prolongation and serious ventricular arrhythmia including torsades de pointes. ${ }^{2-6)}$ Studies with animal myocardium have demonstrated that these drugs are able to prolong action potential duration (APD) through inhibition of the rapid component of the delayed rectifier potassium current (IKr). ${ }^{2,7,8)}$ Since then, the assessment of the risks incurred with noncardiovascular therapeutic agents for cardiac function has received great attention. Some researchers and organizations have postulated that analysis of APD in rabbit Purkinje fibers is the most adequate method for evaluation. ${ }^{9)}$ This appeared reasonable because rabbit Purkinje fibers had been shown to be highly sensitive to drugs with APD-prolonging activity. ${ }^{9-14)}$

On the other hand, we have previously examined the effect of terfenadine on the action potential of isolated guinea pig ventricular tissue and found that the drug reduces the maximum rate of rise $\left(+\dot{V}_{\max }\right)$ but does not prolong APD. ${ }^{15)}$ This was apparently contradictory to the observation that terfenadine prolongs QT interval on electrocardiogram and raised the possibility that the in vivo arrhythmogenic activity of the drug could not be evaluated in isolated tissue preparations from guinea pig ventricle and dog Purkinje fibers. Thus, in the present study, we applied terfenadine to myocardial tissue preparations from various regions of the rabbit heart including the Purkinje fibers to examine the possible APD-prolonging effect of the drug.

\section{MATERIALS AND METHODS}

Microelectrode Recording of Action Potential Configuration Microelectrode experiments were performed as described previously. ${ }^{14-16)}$ Male Japanese white rabbits weigh- ing 2.0 to $2.5 \mathrm{~kg}$ were anesthetized with intravenous pentobarbital sodium $(40 \mathrm{mg} / \mathrm{kg})$. The free running Purkinje fibers, right ventricular free wall, left atrium, and right atrial tissue including sinoatrial node region were quickly dissected from the hearts and placed in an organ bath containing modified physiological salt solution of the following composition (mM): $\mathrm{NaCl} 135, \mathrm{KCl} 5, \mathrm{CaCl}_{2} 2, \mathrm{MgCl}_{2} 1, \mathrm{NaHCO}_{3} 15$, and glucose 5.5 (gassed with $95 \% \mathrm{O}_{2} / 5 \% \mathrm{CO}_{2}, \mathrm{pH} 7.4,37^{\circ} \mathrm{C}$ ). Purkinje fibers, right ventricular muscles, and left atria were driven by external electrical stimulation with bipolar platinum electrodes and rectangular current pulses (5-ms duration, about $1.2 \times$ threshold strength) at a constant frequency $(1 \mathrm{~Hz})$ generated by an electronic stimulator (Nihon Kohden, SEN-3201). Conventional microelectrode penetrations were made with glass microelectrodes filled with $3 \mathrm{~m} \mathrm{KCl}$. The output of a microelectrode amplifier (Nihon Kohden, MEZ8201) was monitored through a dual-beam cathode-ray oscilloscope (Nihon Kohden, VC-11) and fed into a waveform analysis system (DSS98-type IV; Canopus). Drugs were added after preparations were incubated for more than $40 \mathrm{~min}$, after which the action potentials of the preparations were stable. Drug solutions were prepared immediately before the start of the experiments. Terfenadine and cisapride were dissolved in DMSO. A small aliquot of the drug solution was added to the bath to give the desired final concentrations. DMSO alone up to $1.0 \%$, which was the highest concentration reached during the addition of drugs, had no effect on the parameters measured. The significance of difference between means was evaluated using the paired $t$-test. A $p$ value less than 0.05 was considered statistically significant.

Cloning of Human Ether a Go-Go-Related Gene cDNA and Stable Expression in HEK293 Cells Human ether a go-go-related gene (HERG) cDNA fragments were amplified using PCR from a human heart cDNA library (Takara Shuzo Co., Ltd.) with oligonucleotide primers designed based on the published HERG cDNA sequence ${ }^{17)}$ (GenBank accession 
number U04270). The cDNA fragments obtained were assembled with standard ligation techniques and the full-length HERG cDNA was inserted into the pIRES2-EGFP expression vector (Clontech). The expression vector with HERG cDNA was introduced into cultured HEK293 cells with Lipofectamine (Gibco BRL), and stable transformants were obtained by clone culture in the presence of $400 \mu \mathrm{g} / \mathrm{ml} \mathrm{G} 418$ (Geneticin, Gibco BRL). Transformed HEK293 cell clones were observed at an excitation wavelength of $488 \mathrm{~nm}$ and HERG currents were mesured as described below. Among the EGFP fluorescence-positive clones expressing the HERG current, a clone with a current amplitude of about $0.5 \mathrm{nA}$ was chosen for analysis.

Electrophysiologic Recording of Expressed HERG Current HEK293 cells expressing HERG were plated on glass coverslips 48 to $72 \mathrm{~h}$ before electrophysiologic experiments. Whole-cell voltage-clamp experiments were performed in a chamber mounted on the stage of an inverted microscope perfused continuously at a flow rate of 1.0 to $2.0 \mathrm{ml} / \mathrm{min}$. Temperature was maintained at $22-25^{\circ} \mathrm{C}$. The external solution was the same as that used in the microelectrode experiments described above. The patch pipette solution was composed of (in $\mathrm{mM}$ ): $\mathrm{KOH}, 100 ; \mathrm{KCl}, 40$; aspartic acid, 70; $\mathrm{MgCl}_{2}, 1$; ATP-K $\mathrm{K}_{2}, 5$; creatine phosphate- $\mathrm{K}_{2}, 5$; HEPES, 5; and EGTA, 10 (pH 7.2 with $\mathrm{KOH}$ ). Pipette tip resistances were 2 to $4 \mathrm{M} \Omega$ when filled with the patch pipette solution. The amplitude of the steady-state HERG current was measured as the difference between the current at the holding potential of $-80 \mathrm{mV}$ and the current at the end of the depolarizing pulse. The HERG tail current was measured as the peak outward current on repolarization to $-50 \mathrm{mV}$. Data acquisition and analyses were performed with a patchclamp amplifier (Axopatch 1D; Axon Instruments, CA, U.S.A.), a personal computer (Prolinea 486; Compac, TX, U.S.A.) and pCLAMP 5.5.1. software (Axon).

\section{RESULTS}

Effect of Terfenadine on Action Potential of Rabbit Myocardium The action potential parameters of the four regions examined in the absence of drugs were similar to those obtained in earlier studies. ${ }^{14,16,18)}$ In left atrial preparations, terfenadine had no effect on APD at $0.1 \mu \mathrm{M}$ and $1 \mu \mathrm{M}$. At $10 \mu \mathrm{M}$ and $20 \mu \mathrm{M}$, the drug significantly reduced $+\dot{V}_{\max }$ and action potential amplitude, but had no effect on other parameters (Figs. 1Aa, Ba, Table 1). In the Purkinje fibers, terfenadine $20 \mu \mathrm{M}$, significantly decreased action potential amplitude and $+\dot{V}_{\max }$, but had no effect on other parameters including APD (Figs. 1Ab, Bb, Table 1). The percentage of $\mathrm{APD}_{60}(\mathrm{APD}$ at $-60 \mathrm{mV}$ ) was $100.3 \pm 3.1 \%$ in control preparation even at the highest concentration of terfenadine $20 \mu \mathrm{M}$ $(n=4)$. In the ventricle, terfenadine had no effect on action potential parameters at all concentrations examined (Figs. 1Ac, Bc, Table 1). In the sinoatrial node, terfenadine $20 \mu \mathrm{M}$ significantly decreased $+\dot{V}_{\max }$ and increased cycle length, but had no significant effect on other action potential parameters (Fig. 1Ad, Table 2). As terfenadine prolonged APD in none of the four regions examined, we checked the validity of our experiments by testing the effect of cisapride $1 \mu \mathrm{M}$ in each region. Prolongation of APD was observed in all regions as described in our previous report. ${ }^{14)}$
Effect of Terfenadine on HERG Channel Current in HEK293 Cells In whole-cell voltage-clamped HEK293 cells expressing the HERG channel, depolarization to membrane potentials more positive than $-50 \mathrm{mV}$ induced a voltage- and time-dependent outward current (Fig. 2A). A timedependent decrease in outward current was observed at potentials more positive than $0 \mathrm{mV}$ resulting in a bell-shaped current-voltage relationship at steady state (Fig. 2C). On repolarization to $-50 \mathrm{mV}$, an outward tail current was observed. The decay of the tail could be fitted to a single exponential; the time constant $(\tau)$ was $1224 \pm 114 \mathrm{~ms}(n=4$; test potential $+40 \mathrm{mV}$ ). These properties were identical to those of HERG channel currents previously reported. ${ }^{19)}$ Terfenadine $1 \mu \mathrm{M}$ markedly reduced the outward current on depolarization and a time-independent component remained (Fig. 2B). The steady-state outward current amplitude on depolarization to $0 \mathrm{mV}$ in the absence and presence of terfenadine was $0.44 \pm 0.10$ and $0.09 \pm 0.07 \mathrm{nA}(n=4)$, respectively. The terfenadine-sensitive component was also abolished by E4031. The terfenadine-insensitive component was insensitive to E-4031 and was also present in untransfected HEK293 cells. The tail current on repolarization to $-50 \mathrm{mV}$ was completely abolished by terfenadine $1 \mu \mathrm{M}$ (Figs. 2B, D). The peak amplitude of the tail current after a depolarizing pulse to $40 \mathrm{mV}$ was $0.46 \pm 0.05 \mathrm{nA}(n=4)$ in the absence of terfenadine, but was undetectable in its presence (Fig. 2D).

\section{DISCUSSION}

Terfenadine showed no prolongation of the action potential in myocardial tissue preparations from four different regions of the rabbit heart including the Purkinje fibers, which are known to have high sensitivity to drugs with action potentialprolonging activity. ${ }^{9-14}$ ) The maximum concentration of terfenadine examined $(20 \mu \mathrm{M})$ was higher than the maximum clinically approachable plasma concentration of the drug in hepatic compromise or coadministration with drugs affecting its metabolism. ${ }^{20-22)}$ A lack of APD prolongation by terfenadine in tissue has also been reported in guinea pig ventricle, ${ }^{15)}$ and canine, ${ }^{23,24)}$ and porcine Purkinje fiber. ${ }^{24)}$ Thus it appears that terfenadine does not prolong APD in myocardial tissue preparations in general. This contrasts with the observation that terfenadine prolongs APD in isolated myocardial cells. $^{2,25)}$ Our preliminary observation that terfenadine administered through coronary perfusion did not prolong APD suggests that the lack of terfenadine effects on myocardial tissue is not due to limited drug access to the interior region of the preparations. There is a report that terfenadine increased APD by $10 \%$ in Langendorff-perfused guinea pig hearts. ${ }^{26)}$ This might be ascribed to some difference in experimental conditions, but our interpretation is that the net prolongation produced by terfenadine may be negligible or much smaller than those authors' calculation; the time course data presented (Fig. 2 in the previous report ${ }^{26}$ ) indicated that the drug was applied when the APD of the preparation was in a spontaneously increasing phase.

Some reseachers observed that the inhibitory effect of terfenadine when applied from the extracellular side of the cell membrane was weaker than that when applied from the intracellular side. ${ }^{27)}$ This was attributed to the basic nature of terfenadine; the $\mathrm{p} K_{\mathrm{a}}$ of terfenadine is 8.6 , most terfenadine mol- 
A

a

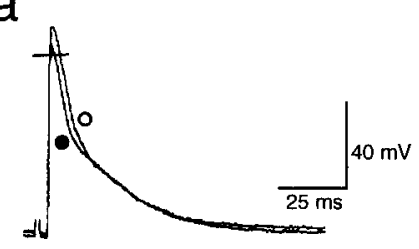

C

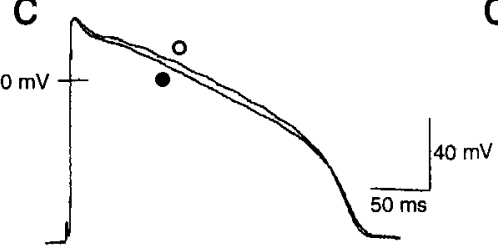

B

a

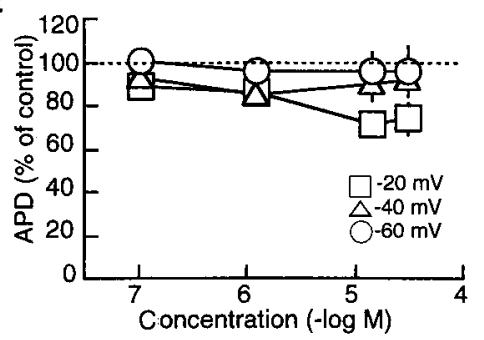

b

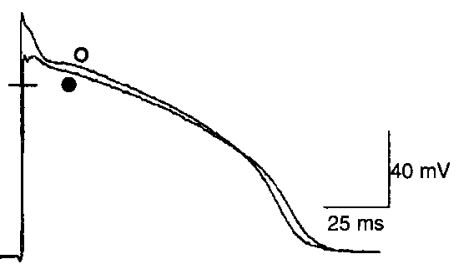

d

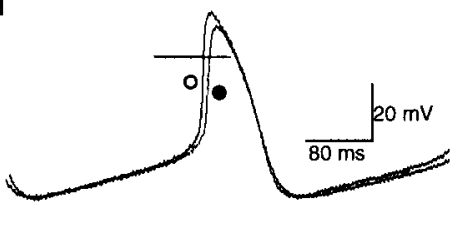

b

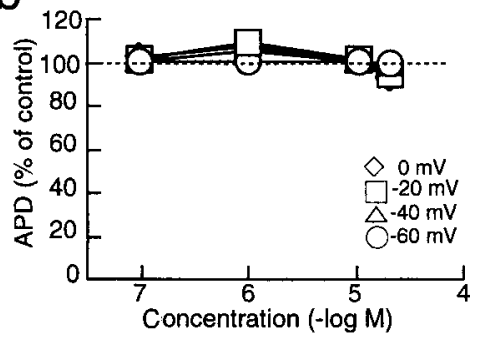

C

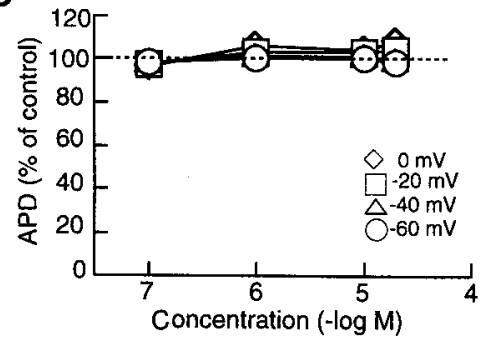

Fig. 1. Effects of Terfenadine on Action Potentials of Isolated Rabbit Myocardial Tissue Preparations

A: Typical recordings of the action potential configuration of the atrium (a), Purkinje fibers (b), ventricle (c), and sinoatrial node (d) obtained in the absence (open circles) and presence (closed circles) of terfenadine $20 \mu \mathrm{M}$. B: Concentration-response curves for the effects of terfenadine on APD of the atrium (a), Purkinje fibers (b), and ventricle (c) at the indicated voltages. Each point with a vertical bar indicates the mean \pm S.E.M. from four to five experiments.

Table 1. Effects of Terfenadine $20 \mu \mathrm{m}$ on Action Potential Parameters of Atrium, Purkinje Fiber, and Ventricular Preparations

\begin{tabular}{|c|c|c|c|c|c|c|}
\hline & \multicolumn{2}{|c|}{ Atrium } & \multicolumn{2}{|c|}{ Purkinje fiber } & \multicolumn{2}{|c|}{ Ventricle } \\
\hline & Before & After & Before & After & Before & After \\
\hline $\mathrm{RP}(\mathrm{mV})$ & $-79.9 \pm 0.5$ & $-73.6 \pm 2.7$ & $-86.5 \pm 1.2$ & $-85.8 \pm 1.1$ & $-82.2 \pm 0.7$ & $-82.4 \pm 0.8$ \\
\hline Amp (mV) & $92.1 \pm 0.7$ & $80.3 \pm 3.0^{*}$ & $114.8 \pm 1.3$ & $104.3 \pm 2.1 *$ & $112.6 \pm 1.4$ & $112.4 \pm 1.0$ \\
\hline $\mathrm{APD}_{0}(\mathrm{~ms})$ & - & - & $61.1 \pm 6.8$ & $53.6 \pm 9.4$ & $84.6 \pm 6.8$ & $92.9 \pm 8.0$ \\
\hline $\mathrm{APD}_{20}(\mathrm{~ms})$ & $8.6 \pm 1.2$ & $7.8 \pm 0.8$ & $104.0 \pm 5.2$ & $102.9 \pm 7.5$ & $142.4 \pm 8.2$ & $149.0 \pm 9.5$ \\
\hline $\mathrm{APD}_{40}(\mathrm{~ms})$ & $16.5 \pm 8.1$ & $18.4 \pm 4.2$ & $139.1 \pm 3.5$ & $139.2 \pm 6.3$ & $186.6 \pm 7.7$ & $186.6 \pm 8.6$ \\
\hline $\mathrm{APD}_{60}(\mathrm{~ms})$ & $46.5 \pm 3.1$ & $50.0 \pm 8.3$ & $156.2 \pm 3.5$ & $156.9 \pm 7.1$ & $205.7 \pm 8.3$ & $202.5 \pm 8.8$ \\
\hline$+\dot{V}_{\max }(\mathrm{V} / \mathrm{s})$ & $214.8 \pm 17.8$ & $161.8 \pm 9.2^{*}$ & $310.0 \pm 35.3$ & $194.8 \pm 12.0 *$ & $191.2 \pm 15.7$ & $188.2 \pm 16.5$ \\
\hline
\end{tabular}

$\mathrm{RP}$, resting potential; Amp, amplitude; $\mathrm{APD}_{0}, \mathrm{APD}_{20}, \mathrm{APD}_{40}, \mathrm{APD}_{60}$, action potential amplitude at $0,-20,-40$, and $-60 \mathrm{mV}$, respectively; repolarization; $+\dot{V}_{\text {max }}$, maximum rate of rise. Values are the mean \pm S.E.M. of four to five experiments. $*$ Significant difference $(p<0.05)$ from corresponding values before the addition of terfenadine. 
A
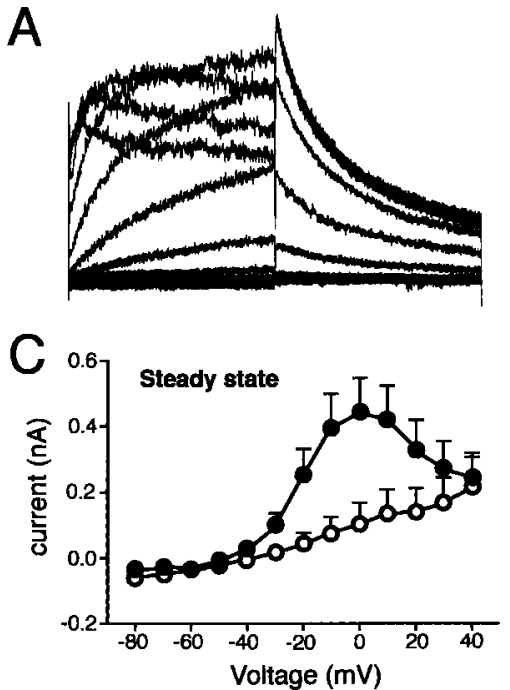

B
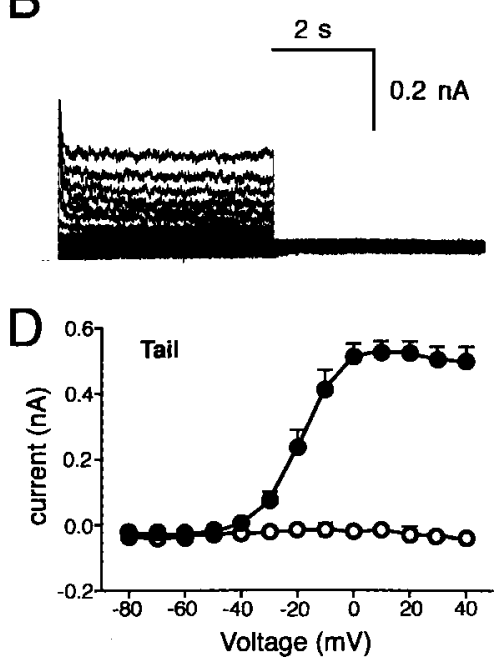

Fig. 2. Effects of Terfenadine on HERG Channel Current Expressed in HEK293 Cells

Voltage clamp pulses $(4 \mathrm{~s})$ to potentials ranging from -80 to $+40 \mathrm{mV}$ in $10 \mathrm{mV}$ increments were applied, followed by a $4 \mathrm{~s}$ step to $-50 \mathrm{mV}$. A and B: Typical current traces before (A) and after (B) the application of terfenadine $1 \mu \mathrm{M}$. C and D: Summarized current-voltage relation in the absence (closed circles) and presence (open circles) of $1 \mu \mathrm{M}$ terfenadine of the steady-state current at the end of the $4 \mathrm{~s}$ test pulse (C) and of the peak tail current during the subsequent step to $-50 \mathrm{mV}$ (D) were plotted versus the voltage of the test pulse. Each point with a vertical bar indicates the mean \pm S.E.M. from four to five experiments.

Table 2. Effects of Terfenadine $20 \mu \mathrm{m}$ on Action Potential Parameters of Rabbit Sinoatrial Node

\begin{tabular}{lrc}
\hline \hline & Before & \multicolumn{1}{c}{ After } \\
\hline MDP $(\mathrm{mV})$ & $-58.6 \pm 0.5$ & $-56.2 \pm 1.2$ \\
OS $(\mathrm{mV})$ & $21.4 \pm 2.3$ & $18.0 \pm 3.0$ \\
Amplitude $(\mathrm{mV})$ & $80.6 \pm 2.9$ & $75.0 \pm 3.9$ \\
$\mathrm{APD}_{50}(\mathrm{~ms})$ & $79.8 \pm 4.7$ & $77.6 \pm 4.9$ \\
$+\dot{V}_{\max }(\mathrm{V} / \mathrm{s})$ & $9.9 \pm 1.7$ & $6.5 \pm 1.8^{*}$ \\
$\mathrm{TH}(\mathrm{mV})$ & $-38.6 \pm 2.3$ & $-33.9 \pm 1.5$ \\
Slope (early; $\mathrm{mV} / \mathrm{s})$ & $74.0 \pm 7.5$ & $70.0 \pm 6.3$ \\
Slope (late; $\mathrm{mV} / \mathrm{s})$ & $116.0 \pm 7.5$ & $104.0 \pm 9.3$ \\
Cycle length $(\mathrm{ms})$ & $353.6 \pm 19.6$ & $389.6 \pm 15.4^{*}$ \\
\hline
\end{tabular}

MDP, maximum diastolic potential; OS, overshoot; $\mathrm{APD}_{50}$, action potential duration at $50 \%$ repolarization; $+\dot{V}_{\max }$, maximum rate of rise; $\mathrm{TH}$, threshold potential. Values are the mean \pm S.E.M. of five experiments. $*$ Significant difference $(p<0.05)$ from corresponding values before the addition of terfenadine.

ecules are charged at the $\mathrm{pH}$ of 7.4 , and thus when applied extracellularly may not be able to approach its binding site close to the cytoplasmic side of the channel protein. To clarify whether this could explain the lack of terfenadine effects on the APD, we applied terfenadine to the HERG current expressed in HEK293 cells in the same extracellular solution as in the microelectrode experiments. Complete inhibition of the HERG current was observed at terfenadine $1 \mu \mathrm{M}$, a concentration much lower than that used in the microelectrode experiments. Thus the lack of terfenadine effects on the APD could not be simply explained by limited accessibility of the drug to its site of action.

The APD prolongation by HERG-inhibiting drugs tends to be more potent at lower stimulation frequencies. ${ }^{228)}$ Inhibition of the HERG channel itself by terfenadine was reported to be unaffected by stimulation frequency in the range of 0.1 to $3 \mathrm{~Hz} .^{28)}$ Thus the frequency dependence of APD prolongation by terfenadine could be ascribed to variation in the contribution of $\mathrm{IKr}$ in repolarization rather than to variations in inhibition of the channel by the drug. ${ }^{29)}$ In isolated ventricular cardiomyocytes, terfenadine $1 \mu \mathrm{M}$ significantly prolonged
APD under a stimulation frequency of $1 \mathrm{~Hz}$. Thus it is likely that a factor(s) other than stimulation frequency is involved in the lack of APD prolongation by terfenadine in the present study.

As multiple ionic currents are involved in the determination of APD, the inhibitory effects of terfenadine on multiple currents might be balanced, resulting in no significant change in APD. Terfenadine is reported to inhibit not only potassium currents but also the sodium and calcium currents. ${ }^{7,30)}$ Also, in the present study, inhibition of $+\dot{V}_{\max }$ was observed in Purkinje fibers and sinoatrial node preparations, indicating that terfenadine has inhibitory effects on these currents in tissue preparations. Among the IKr blockers that prolong APD, E-4031 $1^{31)}$ and dofetilide ${ }^{32)}$ have no effect on sodium and calcium currents. Haloperidol $^{18)}$ and cisapride ${ }^{14)}$ have inhibitory effects on action potential $+\dot{V}_{\max }$, which is an index of the depolarizing sodium current, and their potency is comparable to that of terfenadine. ${ }^{15)}$ Thus, although a systematic quantitative study is necessary for a final conclusion, the lack of APD-prolonging activity of terfenadine in tissue preparations cannot simply be ascribed to its inhibitory effect on sodium and calcium currents at present. Reduction of $+\dot{V}_{\max }$ results in a decrease in conduction velocity and prolongs the time required for propagation of the activation wave front throughout the ventricle and thereby produces asynchrony in the process of activation among various regions of the ventricle. The resulting heterogeneity in repolarization might increase the susceptibility of unidirectional conduction block and reentrant ventricular arrhythmia. However, this does not appear to be the case with terfenadine because the drug is not reported to affect the QRS complex on electrocardiography. Thus the reason for this lack of terfenadine effects on APD in tissue preparations is not clear at present.

Many drugs that are arrhythmogenic and prolong QT interval in vivo prolong APD in isolated myocardial tissue preparations. Purkinje fibers are known to be highly sensitive to the APD-prolonging effect of drugs, ${ }^{9-14)}$ and some researchers have recommended the use of rabbit Purkinje fiber 
action potential for the evaluation of cardiac safety. $\left.{ }^{9}, 10\right)$ The European Federation of Pharmaceutical Industries and Associations has performed a survey on the methodology within the pharmaceutical industry relating to the detection of undesired cardiac effects of active new compounds. ${ }^{33)}$ The most commonly used tissue for in vitro electrophysiology was Purkinje fibers. However, we must be aware that some drugs, such as terfenadine, are arrhythmogenic but do not prolong APD in myocardial tissue preparations. There are other examples of drugs that induce torsades de points and do not prolong APD in tissue preparations such as aprindine ${ }^{34,35)}$ and terodiline. ${ }^{36)}$ Thus electrophysiologic studies in isolated tissue are useful for understanding the mechanisms of arrhythmogenic effects but may not be sufficiently predictive for the purposes of safety pharmacology. ${ }^{37,38)}$

In conclusion, the present results suggest that the HERG channel inhibitory action of certain drugs underlying their in vivo QT prolongation cannot be evaluated based on their action potential-prolonging activity in isolated myocardial tissue preparations.

\section{REFERENCES}

1) Kerin N., Somberg J., Am. Heart J., 128, 575-585 (1994).

2) Salata J., Jurkiewicz N., Wallace A., Stupienski R., Guinosso P., Lynch J., Circ. Res., 76, 110-119 (1995).

3) Zehender M., Hohnloser S., Just H., Cardiovasc. Drugs Ther., 5, 515-530 (1991).

4) Ishii Y., Muraki K., Kurihara A., Imaizumi Y., Watanabe M., Eur. J. Pharmacol., 331, 295-302 (1997).

5) Kodama I., Suzuki R., Kamiya K., Iwata H., Toyama J., Br. J. Pharmacol., 107, 502-509 (1992).

6) Bran S., Murray W., Hirsch I., Palmer J., Arch. Intern. Med., 155, $765-768$ (1995).

7) Tanaka H., Nishimaru K., Sekine T., Shijuku T., Shigenobu K., Res. Comm. Pharm. Toxicol., 2, 163-174 (1997).

8) Eckardt L., Haverkamp W., Borggrefe M., Breithardt G., Cardiovasc. Res., 39, 178-193 (1998).

9) Dumotier B., Adamantidis M., Puisieux F., Bastide M., Dupuis B., Drug Dev. Res., 47, 63-76 (1999).

10) Lu H. R., Marien R., Saels A., De Clerck F., J. Cardiovasc. Electrophysiol., 12, 93-102 (2001).

11) Abrahamsson C., Duker G., Lundberg C., Carlsson L., Cardiovasc. Res., 27, 861-867 (1993).

12) Adamantidis M., Lacroix D., Caron J., Dupuis B., J. Cardiovasc. Phar- macol., 26, 319-327 (1995).

13) Puisieux F., Adamantidis M., Dumotier B.. Dupuis B., Br. J. Pharmacol., 117, 1377-1379 (1996).

14) Noguchi K., Ito C., Isobe Y., Fukushima K., Tanaka Y., Tanaka H., Shigenobu K., Pharmacology, 62, 73-79 (2001).

15) Tanaka H., Masumiya H., Kato Y., Shigenobu K., Gen. Pharmacol., 27, 337-340 (1996).

16) Masumiya H., Tanaka H., Shigenobu K., Eur. J. Pharmacol., 335, 15-21 (1997).

17) Warmke J., Ganetzky B., Proc. Natl. Acad. Sci. U.S.A., 91, $3438-$ 3442 (1994).

18) Noguchi K., Karasawa Y., Isobe Y., Fukushima K., Tanaka H., Shigenobu K., Res. Comm. Biol. Psychol. Psychiat., 24, 21-33 (1999).

19) Katayama Y., Fujita A., Ohe T., Findlay I., Kurachi Y., J. Pharmacol. Exp. Ther, 294, 339-346 (2000).

20) Davies A. J., Harindra V., McEwan A., Ghose R. R., BMJ, 298, 325 (1989).

21) Monahan B. P., Ferguson C. L., Killeavy E. S., Lloyd B. K., Troy J., Cantilena L. R., Jr., JAMA, 264, 2788-2790 (1990).

22) Honig P. K., Wortham D. C., Zamani K., Conner D. P., Mullin J. C., Cantilena L. R., JAMA, 269, 1513-1518 (1993).

23) Lang D., Wang C., Wegener T., J. Cardiovasc. Pharmacol., 22, 432442 (1993).

24) Gintant G., Limberis J., McDermott J., Wegner C., Cox B., J. Cardiovasc. Pharmacol., 37, 607-618 (2001).

25) Kato Y., Mori T., Ohmori K., Ichimura M., Jpn. J. Pharmacol., 70, 199-202 (1996).

26) Pinney S. P., Koller B. S., Franz M. R., Woosley R. L., J. Cardiovasc. Phamacol., 25, 30-34 (1995).

27) Nishio M., Habuchi Y., Tanaka H., Morikawa J., Yamamoto T., Kashima K., J. Pharmacol. Exp. Ther., 287, 293-300 (1998).

28) Crumb W. J., J. Pharmacol. Exp. Ther., 292, 261-264 (2000).

29) Weirich J., Antoni H., Basic Res. Cardiol., 93 (Suppl. 1), 125-132 (1998).

30) Ming Z., Nordin C., J. Cardiovasc. Pharmacol., 26, 761-769 (1995).

31) Wettwer E., Scholtysik G., Schaad A., Himmel H., Ravens U., J. Cardiovasc. Pharmacol., 17 480-487 (1991).

32) Gwilt M., Arrowsmith J. E., Blackburn K. J., Bruges R. A., Cross P. E., Dalrymple H. W., Higgins A. J., J. Pharmacol. Exp. Ther., 256, 318-324 (1991).

33) Reden J., EFPIA/ABPI survey on non-clinical in vivo and in vitro ECG data, 1999, pp. $1-37$.

34) Verdonck F., Vereecke J., Vleugels A., Eur. J. Pharmacol., 26, 338 347 (1974).

35) Steinberg M., Greenspan K., Cardiovasc. Res., 10, 236-244 (1976).

36) Pressler M., Warner M., Rubart M., Rardon D., Zipes D., J. Cardiovasc. Electrophysiol., 6, $443-454$ (1995).

37) Pourrias B., Porsolt R., Lacroix P., Drug Dev. Res., 47, 55-62 (1999).

38) Picard S., Lacroix P., Curr. Opin. Invest. Drugs, 4, 303-308 (2003). 